\title{
Etude comparative de deux écosystèmes forestiers feuillus et résineux des Ardennes primaires françaises
}

\section{III. - Minéralomasse et cycle biologique}

\author{
C. NYS, Dominique RANGER, J. RANGER \\ avec la collaboration technique de P. Bonnaud, D. Gelhaye, Jitka Lhomme, \\ Louisette MaSar et Dominique VaIRelles \\ I.N.R.A., Station de Recherches sur les Sols forestiers et la Fertilisation \\ Centre de Recherches forestières, Champenoux, F 54280 Seichamps
}

\section{Résumé}

L'étude porte sur la comparaison des minéralomasses et du cycle des bioéléments dans deux écosystèmes : un taillis-sous-futaie appauvri (réserves de 150 ans, taillis de 30 ans) et une pessière d'une cinquantaine d'années.

Les principaux résultats concernent :

\section{La méthodologie}

Une certaine rationalisation de l'échantillonnage peut être envisagće à partir des résultats des comparaisons (entre compartiments et entre espèces) et des relations avec les paramètres morphologiques qui permettent d'expliquer une partie de la variabilité observée sur les concentrations.

\section{Les évaluations régionales}

sous forme de tarifs de minéralomasse compartimentée reliant pour chaque compartiment la masse de bioéléments à la circonférence à $1,30 \mathrm{~m}$ de l'arbre.

Ces tarifs sont utilisés pour les évaluations à l'hectare. Pour des peuplements semblables, dans le même contexte écologique, il est possible de connaître avec suffisamment de précision l'exportation de bioéléments liée à l'exploitation de tout ou partie de la biomasse.

\section{Le fonctionnement des écosystèmes}

Les principaux flux annuels entre la végétation et le sol sont mesurés. Le prélèvement des peuplements est évalué par la formule simplifiée :

$$
\text { Prélèvement }=\text { immobilisation }+ \text { retour }
$$

Les deux peuplements ont des immobilisations annuelles faibles avec des rendements biologiques (Matière produite/Eléments consommés) très différents.

L'extrapolation des données brutes a une durée plus significative (150 ans c'est-à-dire une révolution de futaie, 3 de taillis d'une part et 2 d'épicéas d'autre part) est effectuée. Les productions et immobilisations, ainsi que le problème de maintien de la fertilité des sols sont discutés. Au total, il ressort que si les immobilisations peuvent être compensées, au moins partiellement, par les apports atmosphériques et l'altération, il convient de ne pas trop solliciter les sols pauvres pour l'exploitation des petits compartiments très consommateurs en bioéléments. 
Les enquêtes régionales (Li: TACon et al., 1970; Levy, 1978; Decourt \& Nrs, 1976) pour les principales essences forestières françaises ont permis l'établissement des normes qualitatives d'assimilation des éléments minéraux.

Elles permettent maintenant de replacer, par rapport à la moycnne régionale, un peuplement nouveau après une simple analyse foliaire. Dans le contexte actuel des besoins en énergie nouvelle, il est envisagé d'exploiter plus intensément les peuplements forestiers (en particulier les taillis).

Cette intensification des récoltes posera des problèmes théoriques et économiques de fertilisation, que seule l'évaluation de la masse des éléments minéraux, fixés ou immobilisés dans la biomasse, ou minéralomasse, permettra de résoudre. En effet, limmobilisation est un terme important de la circulation des éléments minéraux entre le sol et la plante, c'est-à-dire du cycle biologique, et elle caractérise la productivité de l'écosystème, dont le fonctionnement peut s'écrire sous la forme simplifiée :

$$
\text { Prélèvement réel dans le sol = Immobilisation + retour net des partic aériennes }
$$

formule valable pour un peuplement adulte en équilibre, en considérant comme négligeable l'accroissement de la masse feuilles + radicelles, qui, en toute rigueur, s'ajoute à l'immobilisation proprement dite dans la masse ligneuse. De même, le retour net fait abstraction du terme minéralisation des racines mortes (litière racinaire) égale au prélèvement nécessaire à lélaboration de la masse des radicelles, et on aura alors : retour net $=$ chute de litière + pluviolessivats - apports atmosphériques.

Nous appliquerons ces principes à l'étude comparée des minéralomasses et du cycle biologique de deux peuplements forestiers des Ardennes (forêt domaniale de ChâteauRegnault) dont une première étude vient de paraître (RANGER, 1981) : un taillis-sousfutaie (réserves de 150 ans, taillis de 30 ans) et une pessière de 48 ans (classe de productivité : IV), toutes deux situées sur un même sol brun acide, développé dans une formation limoneuse daltération des schistes réviniens.

\section{Principes d'évaluation de la biomasse et de la minéralomasse bases de l'échantillonnage}

Lapplication de la relation précédente (1) du fonctionnement de l'écosystème impose la mesure de l'immobilisation courante correspondant à l'accroissement courant de la biomasse et celle de la restitution par les retombées biologiques.

\subsection{Mesure des retombées biologiques}

Les retombées de litières sont évaluées par des pièges à litières disposés en ligne sous le peuplement ( 5 fois $0,1365 \mathrm{~m}^{2}$ ). De même, le pluviolessivage est recueilli dans des impluviums élémentaires sous peuplements, disposés en ligne ( 5 fois $0,1365 \mathrm{~m}^{2}$ ) et au hasard ( 5 fois $0,0113 \mathrm{~m}^{2}$ ), tandis que les apports par les eaux météoriques sont mesurés par trois pluviomètres situés hors peuplement. Ces dispositifs sont relevés mensuellement depuis trois ans. Le ruissellement des troncs n’a pa été mesuré mais évalué à partir des données bibliographiques. 


\subsection{Principes d'évaluation de l'accroissement courant en biomasse et minéralomasse}

Pour l'évaluation de la biomasse, puis de la minéralomasse, on aura recours à la notion de compartiments et à l'usage de tarifs propres à chaque compartiment.

1.21. Les tarifs de biomasse compartimentée sont considérés comme applicables pendant au moins un court laps de temps pendant lequel on mesure l'accroissement correspondant.

Pour le taillis, on prélève dans la placette de biomasse moyenne, 25 rondelles pour les espèces principales (bouleau et sorbier), dont on lit les chiffres d'accroissement, qu'on appliquera ensuite aussi aux espèces secondaires.

Pour la futaie, l'évaluation est basée sur les chiffres de production courante en biomasse ligneuse totale de Duvigneaud et al. (1972) sur station analogue, pondérés à notre inventaire.

Pour la pessière, la production courante a été évaluée à partir de carottes de sondage à $1,30 \mathrm{~m}$ sur 30 épicéas de la placette expérimentale.

\subsection{Echantillonnage des compartiments}

Nous avons réalisé, à l'occasion de ce travail, une étude de base de la variabilité des distributions des éléments dans les différents compartiments, fondée sur un protocole d'échantillonnage suffisamment serré pour approcher au mieux la composition minérale par compartiment.

L'acquisition des données de base sur la distribution des bioéléments justifie l'échantillonnage réalisé pour cette étude, et permettra de proposer une procédure simplifiée et efficiente pour les travaux ultérieurs.

Le taillis : quatre séries d'échantillons ont été effectuées : une pour les arbres morts (indépendamment de lessence) et trois pour les arbres vivants (une pour les bouleaux, une pour les sorbiers qui sont les deux essences principales, et une pour les essences d'accompagnement, principalement le chêne, appelées « divers »). Dans chaque série, l'échantillonnage est de type stratifié par classe de surface terrière et proportionnel aux fréquences des classes (soit 14 sorbiers, 24 bouleaux, 7 divers et 29 arbres morts). On a distingué les compartiments suivants :

- Le tronc : un échantillon de bois et un échantillon d'écorce ont été préparés à partir de rondelles prélevées chaque mètre sur le trone en respectant la répartition verticale et horizontale des biomasses dans les troncs. Pour ce faire, un secteur de rondelle d'angle constant au centre est découpé sur chaque rondelle d'épaisseur constante.

- Les branches : trois ou quatre branches vivantes ont été analysées par arbre, en séparant feuilles et rameaux, et une branche morte par arbre.

La futaie : une seule réserve (de chêne) a pu être débitée. Nous l'avons échantillonnée selon le protocole de AtTıwil (1962) (cité par Riedacker, 1969) en séparant les mêmes compartiments que pour le taillis.

La pessière : le principe reste le même que pour les feuillus. 23 épicéas ont été débités. Une différence cependant pour les branches : celles-ci ont été, en effet, regrou- 

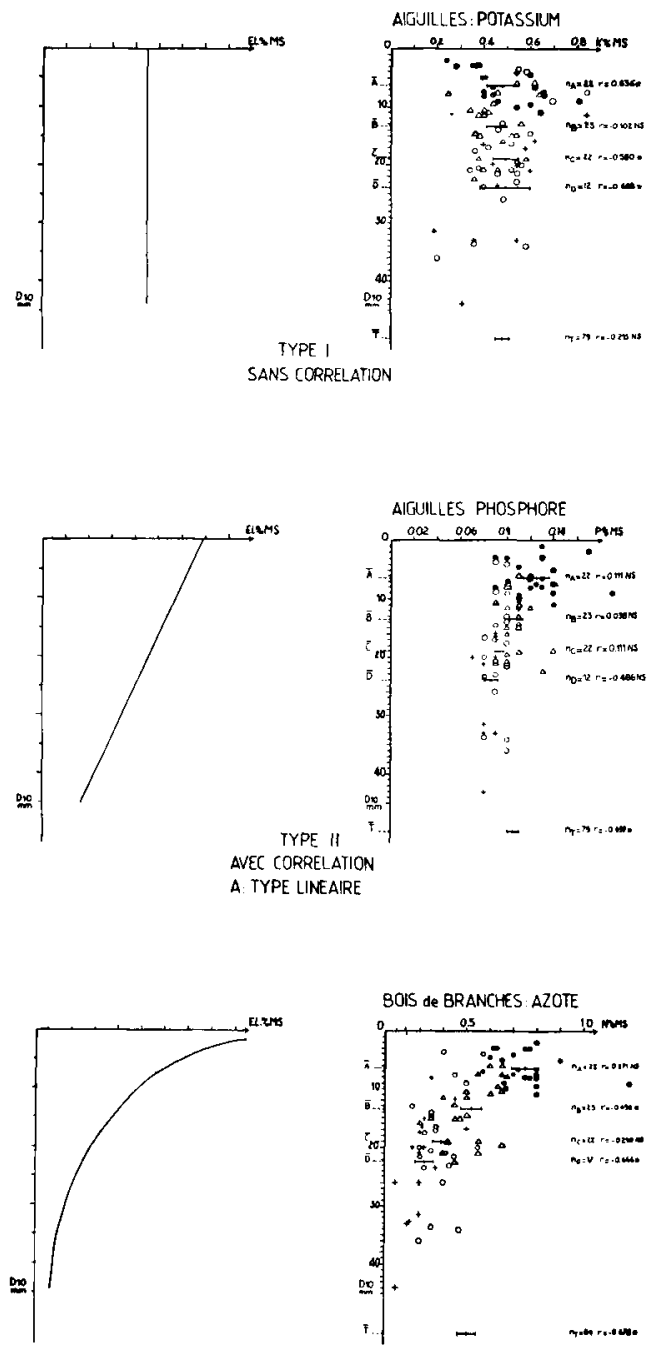

B. TYPE CURMLNEAIRE

Fig. 1

Distribution des bioéléments dans la couronne des épicéas :

- à gauche : distribution théorique; - à droite : exemple.

Bioelement distribution in the spruce crown:

- left : theorical distribution; - right : example.

$\bullet, \triangle, O,+$

$\overrightarrow{\mathrm{A}}, \overline{\mathrm{B}}, \overrightarrow{\mathrm{C}}, \overline{\mathrm{D}}, \overline{\mathrm{T}}$ NA, NB, NC, ND, NT $\mathbf{r}$ *

NS
Respectivement individu des niveaux A, B, C, D.

Diamètre moyen des branches des niveaux A, B, C, D et total.

Nombre d'individus des niveaux A, B, C, D et total.

Coefficient de corrélation.

Coefficient de corrélation significatif au seuil de 5 p. 100.

Coefficient de corrélation non significatif au seuil de 5 p. 100. Intervalle de confiance de la moyenne des individus. 
pées en strates (4 niveaux pour les branches vivantes, 2 niveaux pour les branches mortes). C'est une méthode déjà utilisée par MorRISSON (1972). Une branche a été analysée par niveau.

\subsection{Recherche des lois de distribution des bioéléments dans les compartiments} et évaluation des minéralomasses

Le but de l'analyse par compartiment est d'isoler des organes de composition homogène et dont on recherche les paramètres éventuels de variation. Selon les cas, en effet, il existe ou non une liaison entre concentration minérale dans un échantillon et le ou les paramètres qui ont servi à l'identifier.

Exemple des compartiments aiguilles et branches de l'épicéa : les profils de distribution vertica'e des bioéléments dans la couronne ne dépendent pas de l'arbre, en général (plus de 85 p. 100 des cas), mais principalement de la position dans l'arbre. L'étude des liaisons entre concentrations minérales et le paramètre dendrométrique $D_{10}$ ou le niveau de stratification dans la couronne, montre (fig. 1) plusieurs cas possibles qui guideront l'échantillonnage : absence de corrélation, corrélation linéaire ou curvilinéaire.

En l'absence de corrélation, la concentration du compartiment (par ex. K des aiguilles) est assimilée à la moyenne des concentrations. S'il y a corrélation linéaire, l'échantillonnage du bois des branches, par exemple, peut se faire en fonction du paramètre $\mathrm{D}_{10}$, indépendamment de la hauteur relative de la couronne : c'est le cas du calcium du bois, mais également de $\mathrm{P}, \mathrm{Ca}, \mathrm{Mg}$, des aiguilles, qui ne justifie donc pas de distinguer des niveaux de stratification qu'il faut prendre par contre en considération dans le cas de corrélation curvilénéaire (cas de $\mathrm{N}$ des aiguilles et de NPK des rameaux).

Il faut noter que dans l'étude du compartiment «aiguilles», nous n'avons pas séparé les aiguilles de différents âges, et avons pu ainsi masquer l'effet du paramètre hauteur relative, évoqué par plusieurs auteurs (WELls \& METZ, 1963).

Les profils ainsi obtenus, par le procédé de stratification, sont complexes, mais ils permettent plus aisément que les profils moyens (VAN DEN Driessche, 1974) de saisir les lois de répartition des éléments et de rationaliser l'échantillonnage.

Ainsi par l'interprétation des résultats de l'échantillonnage, on aboutit ou non à l'établissement de relations entre concentrations minérales et paramètres dimensionnels et de situation, qui permettent d'évaluer correctement la concentration des compartiments totaux des arbres échantillonnés.

Le passage au peuplement se fait en plusieurs étapes :

1 : calcul des minéralomasses des compartiments des arbres échantillons;

2 : établissement de tarifs de minéralomasses compartimentées (liaison minéralomasse d'un compartiment $=\mathrm{f} \mathrm{C130)}$;

3 : calcul à partir des tarifs, de la minéralomasse compartimentée des termes médians de chaque classe de surface terrière de l'inventaire et pondération de la fréquence, soit

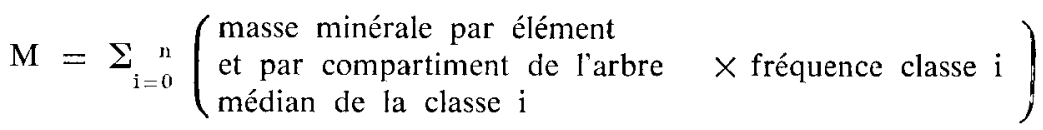


TABleau 1

Composition moyenne de différents compartiments par espèce du taillis et de la futaie.

\begin{tabular}{|c|c|c|c|c|c|}
\hline & & \multicolumn{4}{|c|}{ Taillis* } \\
\hline & & $\mathbf{N}$ & $\mathbf{P}$ & $\mathrm{K}$ & $\mathrm{Ca}$ \\
\hline & $\begin{array}{c}- \text { Bouleau } \\
\mathrm{n}=56\end{array}$ & $\begin{array}{l}1,24^{\mathrm{a}} \quad(0,10) \\
(1) \quad(2) \quad(3)\end{array}$ & $0,20^{\mathfrak{a}}(0,03)$ & $1,01^{\mathrm{a}}(0,19)$ & $0,54^{\mathrm{a}}(0,09)$ \\
\hline \multirow[t]{2}{*}{ Feuilles } & $\begin{array}{c}\text { - Sorbier } \\
\mathbf{n}=50\end{array}$ & $0,98^{\mathrm{b}}(0,09)$ & $0,15^{b}(0,02)$ & $1,50^{\mathrm{b}}(0,21)$ & $0,63^{b}(0,11)$ \\
\hline & $\begin{array}{c}\text { - Divers } \\
n=8\end{array}$ & $1,53^{c}(0,09)$ & $0,29^{c}(0,02)$ & $1,24^{\mathrm{c}} \quad(0,20)$ & $0,38^{e}(0,05)$ \\
\hline \multirow{3}{*}{$\begin{array}{l}\text { Bois }+ \\
\text { écorce des } \\
\text { branches } \\
\text { vivantes }\end{array}$} & $\begin{array}{c}\text { Bouleau } \\
\mathrm{n}=56\end{array}$ & $0,64^{a}(0,13)$ & $0,06^{\mathrm{a}}(0,01)$ & $0,22^{\mathrm{a}}(0,05)$ & $0,32^{\mathrm{a}}(0,07)$ \\
\hline & $\begin{array}{c}- \text { Sorbier } \\
\mathrm{n}=50\end{array}$ & $0,318^{1} \quad(0,09)$ & $0,03^{\prime \prime}(0,01)$ & $0,17 \mathrm{~b}(0,04)$ & $0,3^{a}(0,07)$ \\
\hline & $\begin{array}{c}\text { - Divers } \\
\mathrm{n}=8\end{array}$ & $0,94 \bullet \quad(0,16)$ & $0,07 a \quad(0,02)$ & $0,27^{a}(0,08)$ & $0,25^{\mathrm{b}} \quad(0,03)$ \\
\hline \multicolumn{2}{|c|}{$\begin{array}{l}\text { Branches mortes (toutes } \\
\text { essences confondues) }\end{array}$} & $0,02 \quad(0,04)$ & $0,001(0,002)$ & $0,002 \quad(0,004)$ & $0,01 \quad(0,02)$ \\
\hline \multirow{3}{*}{$\begin{array}{l}\text { Tronc } \\
\text { écorce }\end{array}$} & $\begin{array}{c}\text { - Bouleaut } \\
\mathrm{n}=25\end{array}$ & $0,46^{a} \quad(0,07)$ & $0,04^{a}(0,01)$ & $0,17^{a} \quad(0,03)$ & $0,45^{\mathrm{a}}(0,07)$ \\
\hline & $\begin{array}{c}\text { - Sorbier } \\
n=13\end{array}$ & $0,49^{\mathrm{b}} \quad(0,03)$ & $0,04^{1,}(0,003)$ & $0,28^{\mathrm{b}}(0,04)$ & $0,89^{\mathrm{b}} \quad(0,13)$ \\
\hline & $\begin{array}{c}\text { - Divers } \\
\mathrm{n}=7\end{array}$ & $0,70:(0,08)$ & $0,03^{a}(0,05)$ & $0,21^{\sharp}(0,06)$ & $0,68^{\circ} \quad(0,05)$ \\
\hline \multirow{3}{*}{ Tronc bois } & $\begin{array}{c}\text { - Bouleau } \\
\mathrm{n}=25\end{array}$ & $0,08^{a}(0,02)$ & $0,01^{\mathrm{a}} \quad(\mathbb{E})$ & $0,03^{n}(0,01)$ & $0,07 \mathrm{a}(0,02)$ \\
\hline & $\begin{array}{c}- \text { Sorbier } \\
n=13\end{array}$ & $0,07^{b}(0,01)$ & $0,01^{\mathrm{a}} \quad(\&)$ & $0,05^{1,}(0,02)$ & $0,09^{\prime} \quad(0,03)$ \\
\hline & $\begin{array}{c}- \text { Divers } \\
n=7\end{array}$ & $0,15^{*} \quad(0,02)$ & $0,01^{a} \quad(8)$ & $0,06^{\mathrm{b}}(0,03)$ & $0,11^{b}(0,04)$ \\
\hline \multicolumn{2}{|l|}{$\begin{array}{l}\text { Arbres } \\
\text { morts : }\end{array}$} & $0,20 \quad(0,02)$ & $0,009(0,002)$ & $0,02 \quad(0,01)$ & $0,09 \quad(0,02)$ \\
\hline
\end{tabular}

* Dans chaque colonne figure :

- (1) la valeur moyenne (p. 100 de MS);

- (2) l'exposant $(a, b, c .$.$) qui indique si les valeurs du même compartiment pour chacune$ des espèces sont significativement différentes au seuil de 5 p. 100 (au moins un exposant différent) ou semblables (au moins un exposant commun);

- (3) intervalle de confiance (à 5 p. 100).

** Dans chaque colonne figure le résultat moyen d'analyses (p. 100 de MS). 
La minéralomasse globale du peuplement est la somme pour un même élément de tous les compartiments.

Il est à noter que cette extrapolation à l'hectare, de placettes expérimentales, sans clairière ni chemin de vidange, aboutit en fait, comme pour la biomasse (PARDE 1980) à une évaluation par excès de la minéralomasse.

\subsection{Méthodes analytiques}

Les échantillons végétaux sont séchés à l'étuve à $65^{\circ} \mathrm{C}$, broyés et homogénéisés. L'azote, après minéralisation de type Kjeldahl modifié (acide sulfurique + catalyseur $\mathrm{K}_{2} \mathrm{SO}_{4}, \mathrm{Se}$ ) est déterminé par colorimétrie au bleu d'indophénol sur un autoanalyseur Technicon. La minéralisation en milieu perchlorique, eau oxygénée, permet la détermination de $\mathrm{Ca}, \mathrm{Mg}$ et $\mathrm{Mn}$ par spectrométrie d'absorption atomique, du $\mathrm{K}$ par émission atomique et du $\mathrm{P}$ par colorimétrie du complexe phosphovanadomolybdique sur autoanalyseur Technicon.

Dans les eaux, préalablement filtrées (filtres Whatman GF/A), les éléments Ca, $\mathrm{Mg}, \mathrm{Mn}$ sont dosés directement ainsi que l'azote minéral; le phosphore est analysé après concentration et minéralisation par colorimétrie du complexe phosphosulfomolybdique.

\section{Distribution des bioéléments et calcul de la ninéralomasse}

\subsection{Variabilité des concentrations élémentaires. Tarifs de minéralomassé}

2.11. Le taillis-sous-futaie a donné lieu à une étude complète menée sur les différents compartiments, permettant de mettre en évidence les paramètres dimensionnels spécifiques significatifs de la minéralomasse (base des tarifs établis ensuite conformément au modèle allométrique corrigé : cf. RANGER et al., 1981).

Le tableau 1 montre les variations de composition moyenne $(\mathrm{N}, \mathrm{P}, \mathrm{K}, \mathrm{Ca}, \mathrm{Mg}$, $\mathrm{Mn}$ ) des différents compartiments (feuilles, bois de branches, tronc-écorce et bois) par espèce du taillis et pour la futaie.

Les concentrations foliaires sont 1,5 à 10 fois supérieures à celles de la partic ligneuse ; l'écorce des troncs est de 3 à 10 fois plus riche que le bois. Les différences interspécifiques sont souvent significatives. La variabilité des concentrations d'un compartiment à l'intérieur d'une espèce est importante, mais difficile à expliquer : les relations avec les paramètres dimensionnels (D10 ou C130) ne sont pas toujours significatives.

La tableau 2 propose quelques exemples de tarifs de minéralomasse ayant une bonne valeur prévisionnelle (coefficient de corrélation significatif et résidu faible). En fait, nous disposons de tous les tarifs compartimentés.

Les minéralomasses ont été calculées pour les onze placettes, en valeur absolue (tabl. 3) et relative (tabl. 4). Les variabilités sont du même ordre de grandeur que celles des biomasses. 
2.12. La pessière : les concentrations élémentaires des aiguilles sont 1 à 4 fois plus élevées que celles des rameaux; les teneurs des écorces des troncs sont de 6 à 10 fois plus élevées que celles du bois correspondant dont les teneurs en bioéléments sont toujours très faibles.

Les relations avec les paramètres dimensionnels ont été évoquées pour les branches (\$ 1.23). Pour les écorces des troncs, il existe une relation significative négative entre concentration en $\mathrm{P}, \mathrm{Ca}$ et $\mathrm{Mn}$ et $\mathrm{C} 130$ de larbre. MADGWICK (1964) et VAN DEN Driessche (1974) ont déjà observé que les arbres dont la croissance est la plus faible ont les concentrations les plus élevées.

Le tableau 6 donne les principaux tarifs de minéralomasse utilisés pour évaluer aux tableaux 7 et 8 les minéralomasses compartimentées absolues et relatives de la pessière.

\section{3. Étude du cycle biologique simplifié dans les deux écosystèmes}

La similitude des sols de nos stations, donc de leurs potentialités, est un facteur favorable à la comparaison des deux systèmes. Les âges et les traitements différents le sont par contre beaucoup moins. La comparaison portera dabord sur laccroissement courant et le prélèvement correspondant, puis sur une extrapolation à la durée d'une révolution de futaie (150 ans).

Les productions et accroissements des deux peuplements sont très différents (seul le peuplement définitif est pris en compte). Si l'on se rapporte brutalement à la biomasse ligneuse (en matière sèche) lépicéa produit 70 p. 100 de plus que le taillis-sous-futaie. Ramenées à une biomasse unitaire on constate que les immobilisations sont généralement plus élevées chez les feuillus que chez les résineux (respectivement pour $N, P$, $\mathrm{K}, \mathrm{Ca}, \mathrm{Mg}, \mathrm{Mn}+65$ p. $100,+110$ p. $100,+72$ p. $100,+10$ p. $100,-25$ p. 100 , - 32 p. 100). La même comparaison entre taillis et futaie du taillis-sous-futaie montre que le taillis nest pas plus exigeant que la futaie pour les éléments majeurs. En fait, ces taillis âgés à essences frugales (sorbier et bouleau dominant) exportent peu ; il n'en serait certainement pas de même avec des essences à croissance plus rapide exploitées à courte rotation.

Les retours totaux (litière + pluviolessivage) sont du même ordre de grandeur pour les 2 peuplements si l'on met à part le cas de l'azote qui semble suivre une dynamique particulière dans le taillis-sous-futaie puisqüil y a absorption dans la couronne pendant la période de feuillaison (valeur négative du pluviolessivage net). Ce résultat n’est pas isolé. Miller et al. (1976), Katagiri et Tsutsumi (1978) ont fait la même observation. Le stock d'éléments immobilisés dans les litières est important. Il est au moins deux fois plus é!evé pour $N$ et $P$ sous la pessière, traduisant une vitesse de minéralisation beaucoup plus faible que sous les feuillus.

L.e prélèvement courant actuel est sensiblement le même (sauf pour $N$ ) pour une production différente. Le ren̊lement biologique de l'épicéa est done nettement supérieur à celui du taillis-sous-futaie. Les produits obtenus sont toutefois très différents. Le taillis du taillis-sous-futaie arrivant en fin de révolution est peu productif, et le prélèvement correspondant est donc beaucoup plus faible que celui d'un taillis vigoureux. 
TABLEAU 2

Quelques tarifs de minéralomasse du taillis de taillis-sous-futaie.

\begin{tabular}{|c|c|c|c|c|c|c|c|}
\hline \multicolumn{6}{|c|}{ Bouleau $(n=25)$} & \multicolumn{2}{|r|}{ Sorbi } \\
\hline & & $\mathrm{A}$ & B & $\sigma$ & $\mathrm{r}$ & & $\mathrm{A}$ \\
\hline \multirow{6}{*}{ Tronc total } & $\mathrm{N}$ & $-3,953$ & 2,158 & 0,076 & 0,915 & \multirow{6}{*}{ Tronc total } & $-3,173$ \\
\hline & $\mathrm{P}$ & $-5,435$ & 2,368 & 0.069 & 0,990 & & $-4,533$ \\
\hline & $\mathrm{K}$ & $-4,539$ & 2.231 & 0,078 & 0,985 & & -3.963 \\
\hline & $\mathrm{Ca}$ & $-4,534$ & 2.388 & 0,073 & 0,988 & & -3.113 \\
\hline & $\mathrm{Mg}$ & $-5,172$ & 2.258 & 0,080 & 0,985 & & $-4,452$ \\
\hline & $\mathrm{Mn}$ & -4.855 & 2,269 & 0,069 & 0,989 & & $-3,709$ \\
\hline \multirow{6}{*}{$\begin{array}{l}\text { Arbre total } \\
\text { sans feuille } \\
\text { (partie aćrienne) }\end{array}$} & $\mathrm{N}$ & $-4,233$ & 2.390 & 0.095 & 0,981 & \multirow{6}{*}{$\begin{array}{l}\text { Arbre total } \\
\text { sans feuille } \\
\text { (partie } \\
\text { aérienne) }\end{array}$} & $-3,461$ \\
\hline & $\mathbf{P}$ & $-5,576$ & 2,528 & 0,100 & 0,981 & & $-4,688$ \\
\hline & K & $-5,098$ & 2,567 & 0,087 & 0,986 & & $-4,100$ \\
\hline & $\mathrm{Ca}$ & $-4,638$ & 2,500 & 0,074 & 0,989 & & $-3,347$ \\
\hline & $\mathrm{Mg}$ & 5,277 & 2,372 & 0,082 & 0,985 & & $-4,515$ \\
\hline & $\mathrm{Mn}$ & $-5,164$ & 2,451 & 0,079 & 0,987 & & $-3,992$ \\
\hline \multirow{6}{*}{$\begin{array}{l}\text { Arbre total } \\
\text { (partie aérienne) }\end{array}$} & $N$ & -4.053 & 2.358 & 0,090 & 0,982 & \multirow{6}{*}{$\begin{array}{l}\text { Arbre total } \\
\text { (partie } \\
\text { aérienne) }\end{array}$} & $-3,498$ \\
\hline & $\mathbf{P}$ & $-5,199$ & 2,433 & 0,110 & 0,976 & & $-4,652$ \\
\hline & $\mathrm{K}$ & $-4,731$ & 2,491 & 0,092 & 0,984 & & $-3,927$ \\
\hline & $\mathrm{Ca}$ & $-4,368$ & 2.416 & 0,071 & 0,989 & & $-3,406$ \\
\hline & $\mathrm{Mg}$ & $-4,707$ & 2,209 & 0.071 & 0,987 & & $-4,522$ \\
\hline & $\mathrm{Mn}$ & $-4,975$ & 2,417 & 0,084 & 0,985 & & $-3,974$ \\
\hline
\end{tabular}

$\mathrm{A}$ et $\mathrm{B}:$ coefficient de régression $\log _{10}[\mathrm{Xg}]=\mathrm{A}+\mathrm{B} \log _{10}\left(\mathrm{C}_{\mathrm{t} 0}\right) \mathrm{mm}$.

$\mathrm{n}$ : nombre d'individus $\mathrm{n}=25, \alpha=0,05, \mathrm{r}=0,400$;

$\mathrm{n}=14, \alpha=0,05, \mathrm{r}=0,514$

$\sigma$ : écart type résiduel.

$\mathrm{n}=7, \quad \mathrm{a}=0,05, \mathrm{r}=0,707$.

$r$ : coefficient de corrélation. 
Some bioelements content tables for coppice of the coppice with standards.

\begin{tabular}{|c|c|c|c|c|c|c|c|}
\hline \multicolumn{3}{|l|}{$=14)$} & \multicolumn{5}{|c|}{ Divers $(\mathrm{n}=7)$} \\
\hline B & $\sigma$ & $\mathrm{r}$ & & A & B & $\sigma$ & $r$ \\
\hline 1,804 & 0,077 & 0,977 & \multirow{6}{*}{ Tronc total } & $-3,604$ & 2,052 & 0,100 & 0,972 \\
\hline 1,993 & 0,079 & 0,980 & & $-4,487$ & 1,999 & 0,134 & 0,949 \\
\hline 2.081 & 0,132 & 0,951 & & $-4,388$ & 2,120 & 0,176 & 0,925 \\
\hline 1,873 & 0,066 & 0,984 & & $-4,135$ & 2,367 & 0,114 & 0,973 \\
\hline 2,069 & 0,099 & 0,971 & & -5.659 & 2.487 & 0,136 & 0,965 \\
\hline 1,841 & 0,085 & 0,973 & & $-5,233$ & 2.649 & 0,216 & 0,927 \\
\hline $2,05.1$ & 0,062 & 0,988 & \multirow{6}{*}{$\begin{array}{l}\text { Arbre total } \\
\text { sans feuille } \\
\text { (partie aérienne) }\end{array}$} & $-3,679$ & $2.12 \mathrm{l}$ & 0,081 & 0,982 \\
\hline 2,155 & 0,067 & 0,987 & & $-4,405$ & 2,000 & 0.110 & 0,965 \\
\hline 2,231 & 0,097 & 0,976 & & $-4,100$ & 2.046 & 0.168 & 0,926 \\
\hline 2,050 & 0,057 & 0,990 & & -3.885 & 2.329 & 0.077 & 0,987 \\
\hline 2,175 & 0,060 & 0,990 & & -5.620 & 2.560 & 0,164 & 0,953 \\
\hline 2,052 & 0,070 & 0,985 & & $-4,697$ & 2,468 & 0,182 & 0,939 \\
\hline 2,104 & 0,066 & 0.987 & \multirow{6}{*}{$\begin{array}{l}\text { Arbre total } \\
\text { (partie aérienne) }\end{array}$} & $-3,765$ & 2.176 & 0,073 & 0,987 \\
\hline 2,191 & 0,064 & 0,989 & & $-4,998$ & 2,344 & 0,122 & 0,968 \\
\hline 2,234 & 0,089 & 0,980 & & $-4,168$ & 2,097 & 0.159 & 0,936 \\
\hline 2,101 & 0,073 & 0.985 & & $-3,899$ & 2,360 & 0,069 & 0,990 \\
\hline 2,227 & 0,065 & 0,989 & & -4.958 & 2.315 & 0,089 & 0.982 \\
\hline 2,071 & 0,072 & 0.985 & & $-4,39.1$ & 2.368 & 0.169 & 0,943 \\
\hline
\end{tabular}

A and B : Regression coefficiems of the equations.

$\mathrm{n}:$ Number of trees.

o: Residual standard deviation.

$\mathrm{r}$ : Correlation coefficient. 


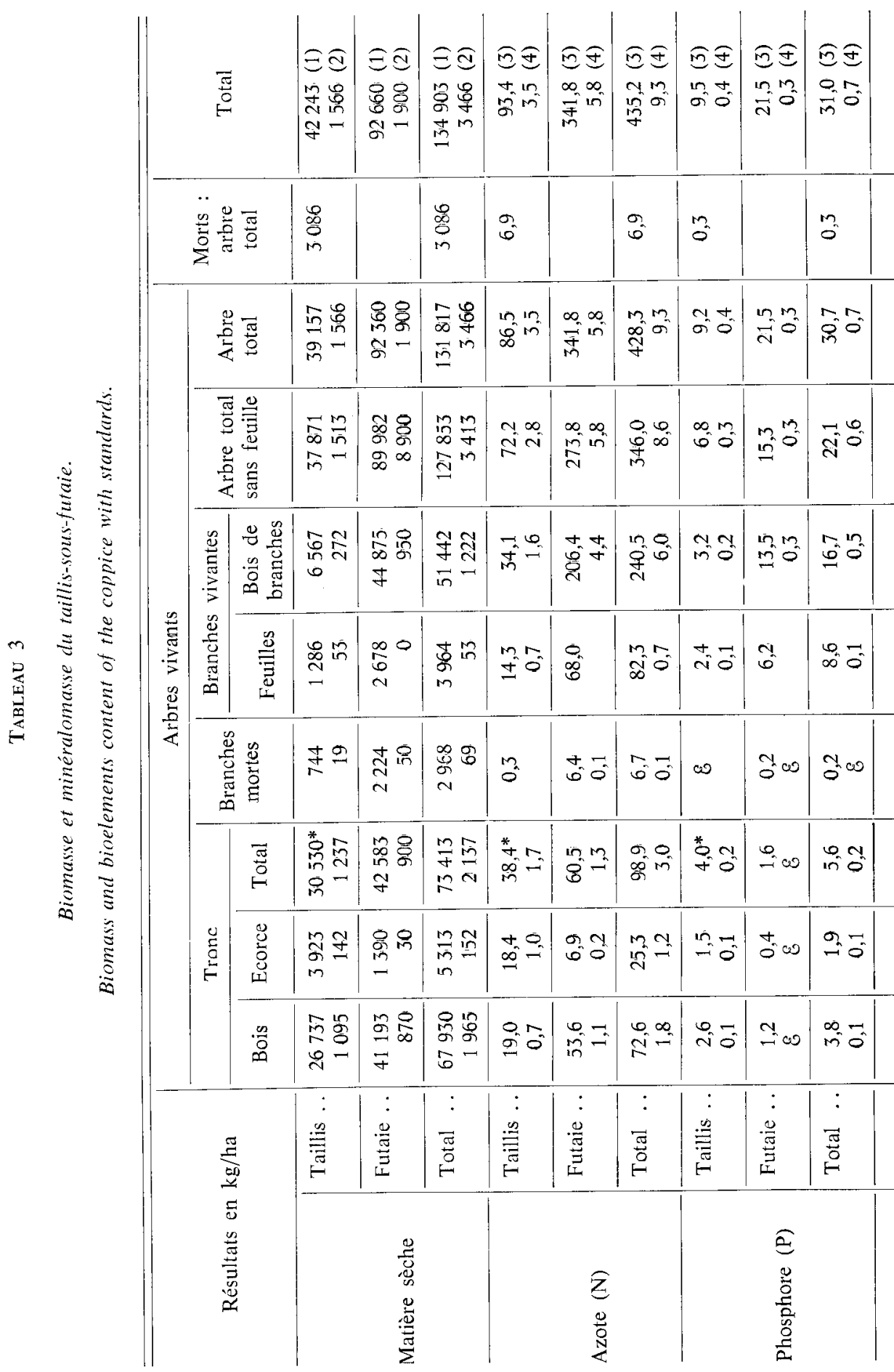




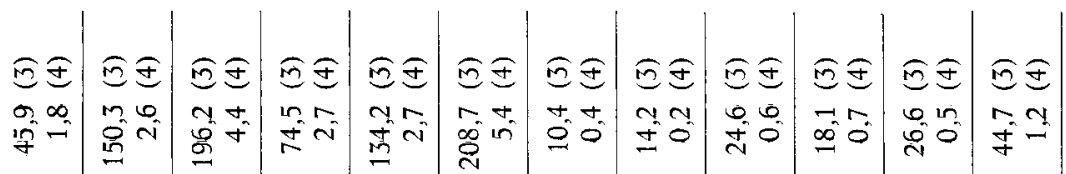

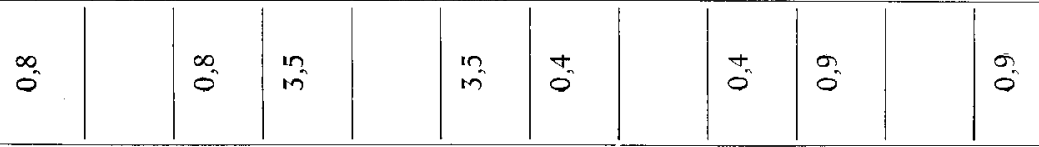

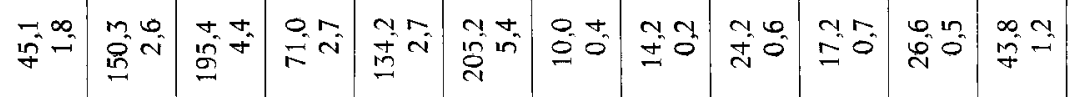

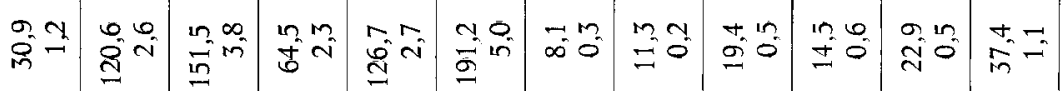

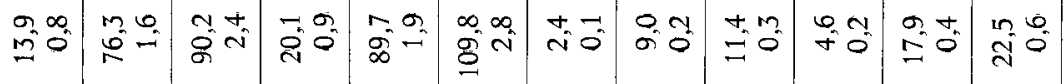

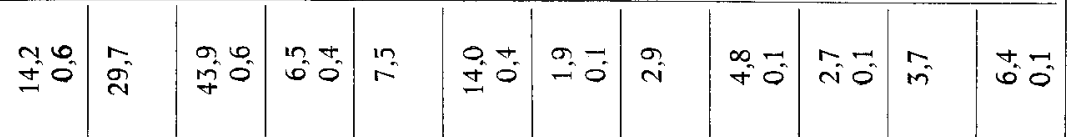

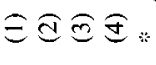

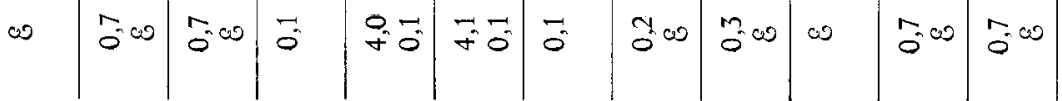

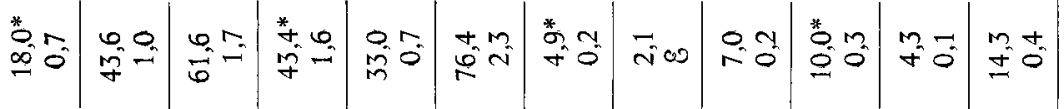

일

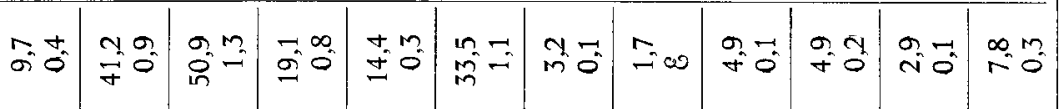

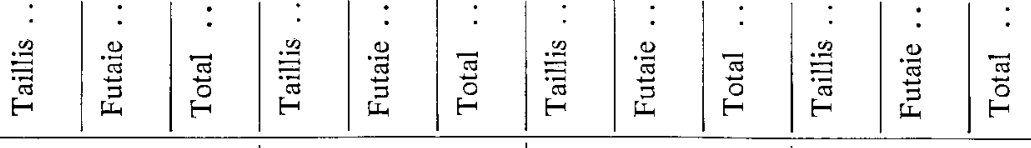

\begin{tabular}{|c|c|c|c|}
\hline $\begin{array}{l}2 \\
a\end{array}$ & 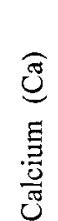 & 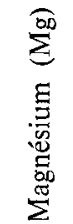 & 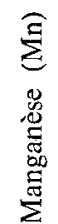 \\
\hline
\end{tabular}

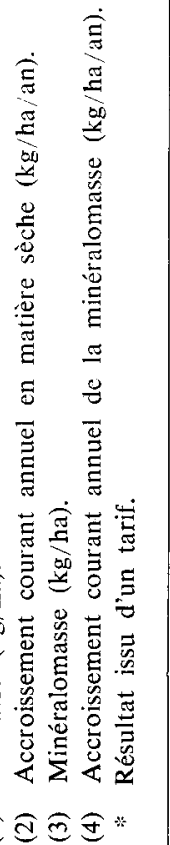


TABleau 4

Distribution relative des biomasse et minéralomasse dans le taillis-sous-futaie.

Relative distribution for biomass and bioelements content of the coppice with standards.

\begin{tabular}{|c|c|c|c|c|c|c|c|c|}
\hline & & MS & $\mathbf{N}$ & $\mathrm{P}$ & $\mathrm{K}$ & $\mathrm{Ca}$ & $\mathrm{Mg}$ & $\mathrm{Mn}$ \\
\hline Arbre total (partie & aérienne) & 100 & 100 & 100 & 100 & 100 & 100 & 100 \\
\hline \multirow{2}{*}{ Feuilles } & $(1)$ & 3,3 & 16,5 & 25,3 & 30,9 & 9,2 & 20,2 & 15,7 \\
\hline & (2) & 2,9 & 19,9 & 28,8 & 19,8 & 5,6 & 20,4 & 13,9 \\
\hline \multirow{2}{*}{$\begin{array}{l}\text { Bois de branches } \\
\text { (vivantes }+ \text { mortes) }\end{array}$} & & 19 & 708 & 326 & 305 & $30 ?$ & 368 & 360 \\
\hline & & 51,0 & 62,2 & 63,7 & 51,2 & 69,8 & 64,8 & 69,9 \\
\hline \multirow{2}{*}{ Bois de tronc } & (1) & 68,3 & 22,0 & 26,3 & 21,1 & 27,6 & 34,0 & 29,1 \\
\hline & (2) & 44,6 & 15,7 & 5,6 & 27,4 & 10,7 & 12,0 & 10,9 \\
\hline \multirow{2}{*}{ Trone total } & (1) & 78,0 & 43,3 & 42,1 & 38,5 & 61,6 & 53,1 & 57,6 \\
\hline & (2) & 46,1 & 17,7 & 7,4 & 29,0 & 24,6 & 14,8 & 16,2 \\
\hline
\end{tabular}

(1) Taillis - Coppice

(2) Futaie - Standards.

Il est d'autre part nécessaire de ne pas juger le rendement biologique du taillis à partir des seules données mesurables : matière sèche et bioéléments, mais aussi en tenant compte du facteur difficilement mesurable qu'est l'âge des souches. Les apports externes sont importants (région industrielle) et couvrent les besoins actuels des peuplements (ULRICH, 1973 ; BUNN \& WILL, 1973).

\subsection{Extrapolation à la durée d'une révolution de futaie feuillue}

Par extrapolation nous avons évalué l'immobilisation comparée dans les deux peuplements, pendant une unité de temps biologiquement significative. Des durées de rotation de 150 ans pour la futaie, de 30 ans pour le taillis et de 75 ans pour la pessière ont été retenues.

Nous posons les hypothèses de travail suivantes :

- les arbres de futaie résultent d'un balivage dès la première rotation de taillis ;

- le taillis n'exporte que les produits de la coupe définitive ; 


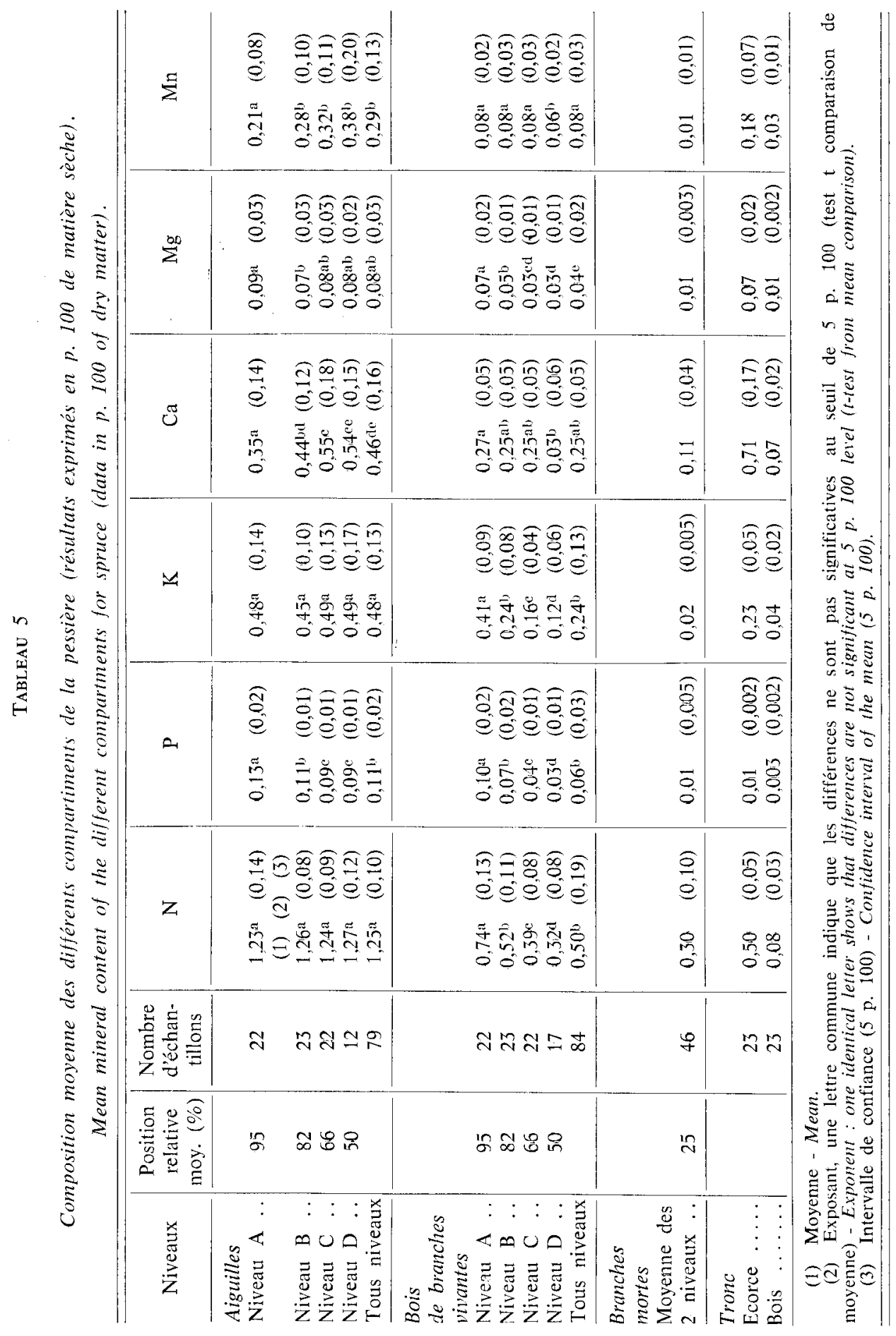


Tableau 6

Tarifs de minéralomasse de la pessière.

\begin{tabular}{|c|c|c|c|c|c|c|c|}
\hline & & A & B & $\sigma$ & $\mathrm{r}$ & & A \\
\hline \multirow{6}{*}{$\begin{array}{c}\text { Aiguilles } \\
n=23\end{array}$} & $N$ & -4.169 & 2.311 & 0,146 & 0.923 & \multirow{6}{*}{$\begin{array}{c}\text { Branches } \\
\text { vivantes } \\
\text { Bois }+ \text { écorce } \\
n=23\end{array}$} & $-5,506$ \\
\hline & P $\ldots$ & $-4,984$ & 2.213 & 0,122 & 0.940 & & $-6,758$ \\
\hline & $\mathrm{K}$ & $-4,540$ & 2.285 & 0,200 & 0.866 & & - 6,668 \\
\hline & $\mathrm{Ca}$ & $-4,778$ & 2,378 & 0,182 & 0,892 & & $-6,149$ \\
\hline & $\mathrm{Mg} .$. & $-4,410$ & 1,955 & 0,175 & 0,861 & & $-7,636$ \\
\hline & Mn ... & $-4,990$ & 2,371 & 0,215 & 0,858 & & - 7,297 \\
\hline \multirow{6}{*}{$\begin{array}{c}\text { Tronc écorce } \\
n=23\end{array}$} & $\mathrm{~N}$ & -4.739 & 2,291 & 0,090 & 0,958 & \multirow{6}{*}{$\begin{array}{c}\text { Tronc bois } \\
\mathrm{n}=23\end{array}$} & - 4,887 \\
\hline & $\mathbf{P}$. & -7.137 & 2,528 & - & 0,926 & & \\
\hline & $\mathrm{K}$. & $--5,057$ & 2,278 & 0,135 & 0,931 & & $-7,640$ \\
\hline & $\mathrm{Ca} \ldots$ & -3.182 & 1,777 & 0.099 & 0,939 & & $-4,628$ \\
\hline & $\mathrm{Mg} \ldots$ & $-5,163$ & 2,123 & 0,119 & 0,938 & & $-6,235$ \\
\hline & Mn ... & $-3,945$ & 1,836 & 0,167 & 0,858 & & $-5,551$ \\
\hline \multirow{6}{*}{$\begin{array}{c}\text { Arbre vivant } \\
\text { total } \\
n=23\end{array}$} & $\mathrm{~N}$ & $-3,698$ & 2,261 & 0,107 & 0.955 & \multirow{6}{*}{$\begin{array}{c}\text { Arbre mort } \\
\text { total } \\
n=10\end{array}$} & $-4,632$ \\
\hline & $\mathrm{P}$ & $-5,462$ & 2,465 & 0,145 & 0,937 & & $-10,040$ \\
\hline & $\mathrm{K}$ & $-5,229$ & 2,662 & 0,181 & $0,9 \cdot 13$ & & $-6,537$ \\
\hline & $\mathrm{Ca} \ldots$ & $-3,881$ & 2,234 & 0.102 & 0,958 & & $-6,561$ \\
\hline & Mg ... & $-5,010$ & 2,349 & 0,119 & 0,948 & & - 3,198 \\
\hline & $\mathrm{Mn} \ldots$ & $-4,750$ & 2,417 & 0,161 & 0,915 & & $-9,963$ \\
\hline
\end{tabular}

$\mathrm{A}$ et $\mathrm{B}$ : Coefficients de régression, $\log _{\omega}[\mathrm{Xg}]=\mathrm{A}+\mathrm{B} \log _{11}\left(\mathrm{C}_{3}\right) \mathrm{mm}$.

$\sigma:$ Ecart type résiduel.

$r$ : Coefficient de corrélation.

$\mathrm{n}$ : Nombre d'individus, $\mathrm{n}=23, \alpha=0,05, \mathrm{r}=0,400$;

$\mathrm{n}=10, a=0,05, \mathrm{r}=0,602$. 
Bioelements content tables for the spruce stand.

\begin{tabular}{|c|c|c|c|c|c|c|c|}
\hline B & $\sigma$ & $r$ & & A & B & $\sigma$ & $r$ \\
\hline 2,648 & 0,150 & 0,937 & \multirow{6}{*}{$\begin{array}{c}\text { Branches mortes } \\
\text { Bois }+ \text { écorce } \\
n=23\end{array}$} & $-0,305$ & 0,593 & 0.154 & 0,504 \\
\hline 2,758 & 0,153 & 0,939 & & $-2,028$ & 0.740 & 0,220 & 0,454 \\
\hline 2,933 & 0,188 & 0.921 & & $-2,619$ & 0,982 & 0,209 & 0,580 \\
\hline 2,800 & 0,141 & 0.949 & & $-1,752$ & 0,958 & 0.214 & 0,561 \\
\hline 3,034 & 0,188 & 0,926 & & $-3,512$ & $1,16.1$ & 0,193 & 0,675 \\
\hline 3,024 & 0,185 & 0.928 & & $-5,580$ & 0,914 & 1,648 & 0,313 \\
\hline 2,398 & 0,124 & 0,946 & \multirow{6}{*}{$\begin{array}{c}\text { Trone total } \\
\mathrm{n}=23\end{array}$} & -4.525 & 2,353 & 0,101 & 0,962 \\
\hline & & & & -7.137 & 2.528 & - & 0,926 \\
\hline 3,280 & 0,191 & 0.933 & & $-6,380$ & 2.906 & 0,167 & 0,935 \\
\hline 2,294 & 0,091 & 0,967 & & $-3,593$ & 2.033 & 0,082 & 0,966 \\
\hline 2,595 & 0,087 & 0,976 & & $-5,561$ & 2.425 & 0.091 & 0.971 \\
\hline 2,492 & 0,136 & 0,941 & & $-4,668$ & 2,250 & 0.141 & 0,924 \\
\hline 2,435 & 0,178 & 0,967 & & & & & \\
\hline 4,002 & - & 0,789 & & & & & \\
\hline 2,777 & 0,067 & 0,957 & & & & & \\
\hline 3,107 & 0,041 & 0,980 & & & & & \\
\hline 1,428 & 0,067 & 0,448 & & & & & \\
\hline 4,270 & 0,134 & 0,966 & & & & & \\
\hline
\end{tabular}

$\mathrm{A}$ and $\mathrm{B}:$ Regression coefficients of equations.

$\sigma:$ Residual standard deviation.

$r$ : Correlation coefficient.

$\mathrm{n}$ : Number of trees. 


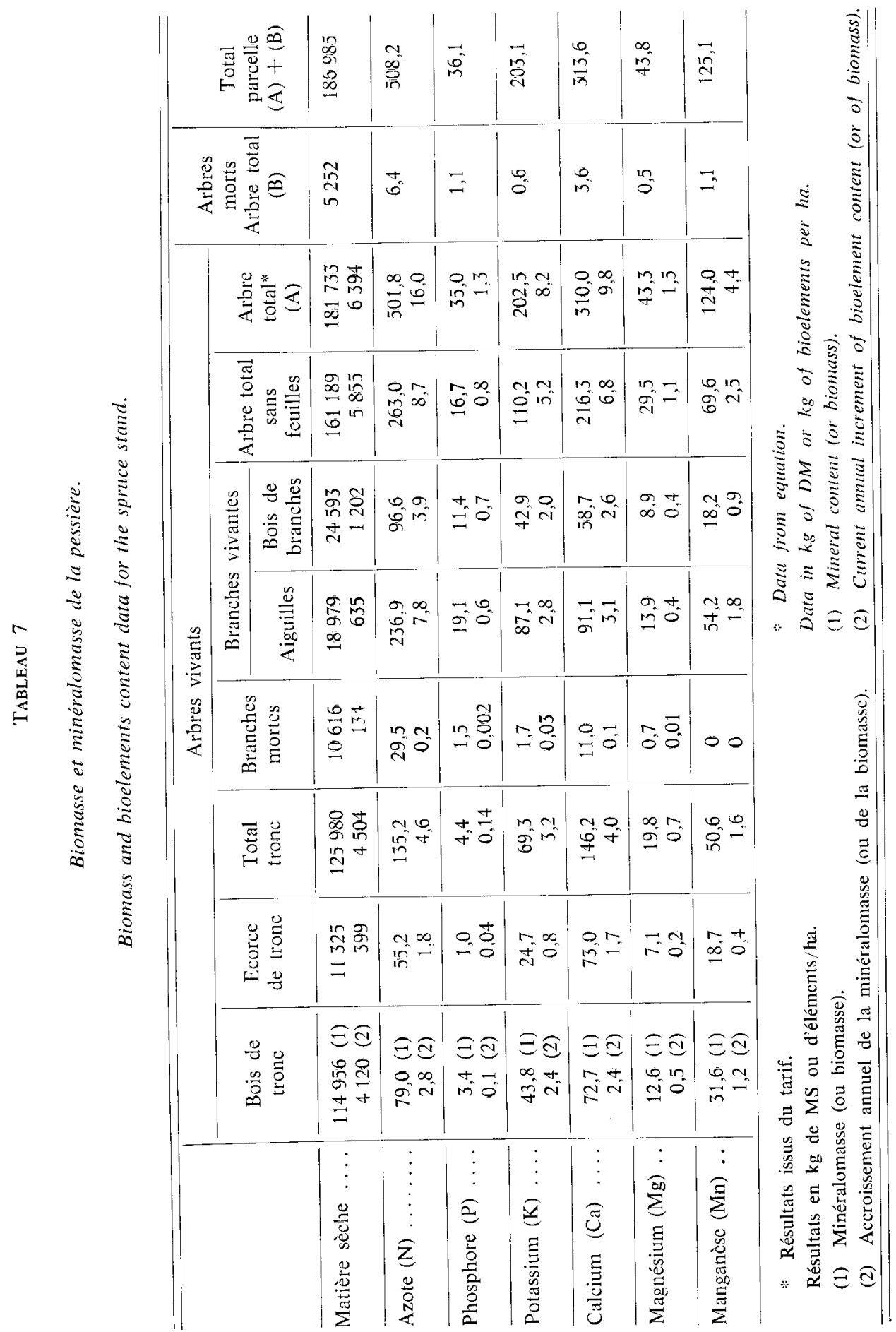




\section{Tableau 8}

Distribution relative des biomasse et minéralomasse dans la pessière.

Relative data for biomass and bioelements content of the spruce stand.

\begin{tabular}{|c|c|c|c|c|c|c|c|}
\hline & MS & $\mathrm{N}$ & $P$ & K & Cá & $\mathrm{Mg}$ & $\mathrm{Mn}$ \\
\hline Arbre total (partie aérienne) & 100 & 100 & 100 & 100 & 100 & 100 & 100 \\
\hline Feuilles & 10 & 48 & 52 & 44 & 30 & 32 & 44 \\
\hline $\begin{array}{l}\text { Bois de branches vivantes }+ \\
\text { mortes } \ldots \ldots \ldots \text {. . . . . }\end{array}$ & 20 & 25 & 35 & 22 & 23 & 22 & 15 \\
\hline Bois de tronc & 64 & 16 & 9 & 22 & 24 & 29 & 26 \\
\hline Trone total & 70 & 27 & 12 & 34 & 47 & 46 & 41 \\
\hline
\end{tabular}

- la pessière na pas été éclaircie jusqu'à 45 ans (sa biomasse à 75 ans est évaluée en consioérant que le tronc double sa biomasse et que les branches ont un accroissement de 10 p. 100 de leur masse entre 45 et 75 ans);

- l'immobilisation dans les parties ligneuses est constante pour les 5 rotations du taillis et les 2 rotations d'épicéas.

Les résultats de ce calcul sont consignés dans le tableau 10.

La production de la pessière est double de celle du taillis-sous-futaic, pour des prélèvements en éléments minéraux voisins, sauf pour les ions alcalino-terreux et le manganèse, dont l'exportation par la pessière est au moins égale à deux fois celle observée pour le taillis-sous-futaie. Le rapport MS produite/éléments consommés est généralement favorable à l'épicéa (sauf pour $\mathrm{Mn}$ ).

Si les chiffres d'immobilisation totale (sans les feuilles) sont mis en parallèle avec les quantités d'éléments assimilables présents cans le sol (bilan sur $1 \mathrm{~m}$ ), on constate que l'ordre de grandeur est le même pour $\mathrm{K}, \mathrm{Ca}, \mathrm{Mg}$ et $\mathrm{Mn}$ (une étude précise des réserves nécessiterait la prise en compte de l'état initial du sol au début de la révolution, ce qui ne peut être réalisé). Cette constatation conduit à deux remarques :

1) les peuplements vont moduler leur production en fonction des générations;

2) la définition du compartiment "éléments assimilables» doit être remise en cause. 
Tableau 9

Quelques données du cycle biologique dans les deux peuplements.

\begin{tabular}{|c|c|c|c|c|c|c|}
\hline & \multicolumn{6}{|c|}{$\begin{array}{l}\text { Feuillus } \\
\text { Taillis sous futaie }\end{array}$} \\
\hline & MS & $\mathrm{N}$ & $\mathrm{P}$ & $\mathrm{K}$ & $\mathrm{Ca}$ & $\mathrm{Mg}$ \\
\hline $\begin{array}{l}\text { Immobilisation courante an- } \\
\text { nuelle } \ldots \ldots \ldots \ldots \ldots \ldots \ldots \ldots\end{array}$ & 3413 & 8,6 & 0,6 & 3,8 & 5,0 & 0,5 \\
\hline $\begin{array}{l}\text { Accroissement courant an- } \\
\text { nuel de la minéralomasse fo- } \\
\text { liaire } \ldots \ldots \ldots \ldots \ldots \ldots \ldots\end{array}$ & 53 & 0,7 & 0,1 & 0,6 & 0,4 & 0,1 \\
\hline $\begin{array}{l}\text { Retours : } \\
- \text { Pluviolessivage }(1)\end{array}$ & & & & & & \\
\hline $\begin{array}{l}\text { Lessivage des cimes .... } \\
\text { Ruissellement des troncs }\end{array}$ & & 13,1 & 0,7 & 38,5 & 18,3 & 4,3 \\
\hline & & $\begin{array}{l}(0,7) \\
29,1\end{array}$ & 0,4 & $\begin{array}{c}(1,9) \\
2,9\end{array}$ & $\begin{array}{c}(0,9) \\
6,1\end{array}$ & $\begin{array}{c}(0,2) \\
1,4\end{array}$ \\
\hline & & $-15,4$ & 0,4 & 37,5 & 13,2 & 3,0 \\
\hline - Chute de litière (3) .... & 4724 & 61,0 & 3,1 & 9,1 & 21,7 & 3,1 \\
\hline $\begin{array}{l}\text { Retours totaux nets } \\
(1-2)+3 \ldots \ldots\end{array}$ & & 45,6 & 3,5 & 46,6 & 34,9 & 6,1 \\
\hline $\begin{array}{l}\text { *Prélèvement courant annuel } \\
\text { Immob. }+ \text { retours nets } . . .\end{array}$ & & 54,2 & 4,1 & 50,4 & 39,9 & 6,6 \\
\hline Strate herbacée totale & 670 & 12,5 & 0,9 & 9,2 & 1,7 & 0,7 \\
\hline Stock des litières & 17300 & 234 & 20 & 53 & 66 & 13 \\
\hline $\begin{array}{l}\text { Sol minéral : } \\
\text { - Eléments échangeables ou } \\
\text { assimilables .......... } \\
\text { - Eléments mobilisables ... } \\
\text { - Eléments totaux ....... }\end{array}$ & & $\overline{-}$ & $\frac{510}{4650}$ & $\begin{array}{r}270 \\
1390 \\
228880\end{array}$ & $\frac{370}{11930}$ & $\begin{array}{r}80 \\
970 \\
30290\end{array}$ \\
\hline
\end{tabular}

* Dans le cas des résineux (feuilles persistantes), on doit ajouter $\Delta \mathrm{f}=$ Accroissement courant de la minéralomasse foliaire. 
Some biological cycle data for each stand.

\begin{tabular}{|c|c|c|c|c|c|c|c|}
\hline \multirow[b]{2}{*}{$\mathrm{Mn}$} & \multicolumn{7}{|c|}{$\begin{array}{c}\text { Résineux } \\
\text { Plantation équienne d'épicea }\end{array}$} \\
\hline & MS & $\mathrm{N}$ & $\mathrm{P}$ & $\mathrm{K}$ & $\mathrm{Ca}$ & $\mathrm{Mg}$ & $\mathrm{Mn}_{\mathrm{n}}$ \\
\hline 1,1 & 5855 & 8,7 & 0,8 & 5,2 & 6,8 & 1,1 & 2,5 \\
\hline 0,1 & 635 & 7,8 & 0,6 & 2,8 & 3,1 & 0,4 & 1,8 \\
\hline 6,6 & & 44,2 & 1,0 & 23,8 & 21,0 & 4,1 & 5,8 \\
\hline$(0,3)$ & & $(2,2)$ & $(0,1)$ & $(2,4)$ & $(2,1)$ & $(0,4)$ & $(0,6)$ \\
\hline 0 & & 29,1 & 0,4 & 2,9 & 6,1 & 1,4 & 0 \\
\hline 6,9 & & 17,3 & 0,7 & 23,3 & 17,0 & 3,1 & 6,4 \\
\hline 13,7 & 4673 & 57,5 & 4,0 & 8,9 & 14,7 & 1,9 & 6,7 \\
\hline 20,6 & & 74,8 & 4,7 & 32,2 & 31,7 & 5,0 & 13,1 \\
\hline 21,7 & & 91,3 & 6,1 & 40,2 & 41,6 & 6,5 & 17,4 \\
\hline 1,0 & 0 & 0 & 0 & 0 & 0 & 0 & 0 \\
\hline 25 & 37300 & 663 & 40 & 56 & 90 & 16 & 23 \\
\hline 110 & & - & 540 & 340 & 420 & 110 & 120 \\
\hline 3300 & & - & & 1. 290 & & 970 & 3140 \\
\hline 4500 & & 15900 & 5390 & 250040 & 15430 & 27830 & 4030 \\
\hline
\end{tabular}




\section{TABLEAU 10}

Extrapolation à 150 ans des données de biomasse et minéralomasse des deux peuplements (biomasse en tonne de matière sèche $\cdot \mathrm{ha}^{-1}$; éléments en $\mathrm{kg} \cdot \mathrm{ha}^{-1}$ ).

Extrapolation at 150 years of biomass production and bioelements incorporation for each stand (biomass in metric ton of dry matter. ha ${ }^{-1}$; biselements in $\mathrm{kg}_{\mathrm{g}} \mathrm{ha}^{-1}$ ).

\begin{tabular}{|c|c|c|c|c|c|c|c|}
\hline & MS & $\mathrm{N}$ & $\mathrm{P}$ & $\mathrm{K}$ & $\mathrm{Ca}$ & $\mathrm{Mg}$ & $\mathrm{Mn}$ \\
\hline $\begin{array}{l}\text { Futaie } \\
\qquad \begin{aligned} 1 \text { trone total } \ldots \ldots \\
2 \text { biomasse ligneuse } \\
\text { totale } \ldots \ldots \ldots \ldots\end{aligned}\end{array}$ & $\begin{array}{l}43 \\
90\end{array}$ & $\begin{array}{r}61 \\
274\end{array}$ & $\begin{array}{r}2 \\
15\end{array}$ & $\begin{array}{r}44 \\
121\end{array}$ & $\begin{array}{r}33 \\
127\end{array}$ & $\begin{array}{r}2 \\
11\end{array}$ & $\begin{array}{r}4 \\
23\end{array}$ \\
\hline Taillis $\begin{aligned} & \\
& 1^{\prime} \text { trone total } \ldots . . . \\
& 2, \text { biomasse ligneuse } \\
& \text { totale } \ldots \ldots \ldots .\end{aligned}$ & $\begin{array}{l}153 \\
189\end{array}$ & $\begin{array}{l}192 \\
395\end{array}$ & $\begin{array}{l}20 \\
35\end{array}$ & $\begin{array}{r}90 \\
160\end{array}$ & $\begin{array}{l}217 \\
325\end{array}$ & $\begin{array}{l}25 \\
40\end{array}$ & $\begin{array}{l}50 \\
75\end{array}$ \\
\hline $\begin{array}{l}1+1^{\prime} \ldots \ldots \ldots \ldots \ldots \\
2+2^{\prime} \ldots \ldots \ldots \ldots\end{array}$ & $\begin{array}{l}196 \\
279\end{array}$ & $\begin{array}{l}253 \\
669\end{array}$ & $\begin{array}{l}22 \\
50\end{array}$ & $\begin{array}{l}134 \\
281\end{array}$ & $\begin{array}{l}250 \\
452\end{array}$ & $\begin{array}{l}27 \\
51\end{array}$ & $\begin{array}{l}54 \\
98\end{array}$ \\
\hline 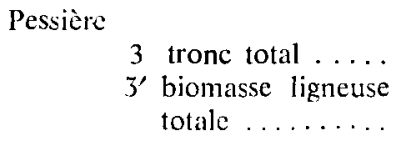 & $\begin{array}{l}516 \\
558\end{array}$ & $\begin{array}{l}554 \\
838\end{array}$ & $\begin{array}{l}41 \\
44\end{array}$ & $\begin{array}{l}284 \\
386\end{array}$ & $\begin{array}{l}600 \\
760\end{array}$ & $\begin{array}{r}82 \\
104\end{array}$ & $\begin{array}{l}208 \\
250\end{array}$ \\
\hline$\frac{\text { Pessière }}{\mathrm{TSF}}=\frac{3}{1+1^{\prime}}$ & 5,4 & 2.2 & 0,2 & 2,1 & 2,4 & 3,0 & 3,9 \\
\hline$\frac{\text { Pessière }}{\text { TSF }}=\frac{3^{\prime}}{2+2^{\prime}}$ & 2 & 1,3 & 0,6 & 1,4 & 1.7 & 2,0 & 2,6 \\
\hline $\begin{array}{c}\text { ES. immob. } \\
\text { Stock assimil. } \\
\text { pessièrc } .\end{array}$ & & & 0,10 & 1,04 & 1,22 & 0,46 & 0,82 \\
\hline
\end{tabular}

On na pas, dans ce cas précis, de preuves absolues quiune deuxième ou troisième génération d'épicéa aurait la même croissance, de même que les rotations du taillis seraient équivalentes; cependant il est peu probable quine perte importante de production ait lieu à moyen terme. Le compartiment «éléments échangeables » est transitoire et susceptible de se recharger par altération comme l'ont montré BonNEAU et al. (1977) et Nys (1981). Quand on raisonne en terme de bilan pondéral des réserves du sol disponibles pour les végétaux, il semble logique de s’adresser à un compartiment moins transitoire du type «éléments mobilisables» bien que non disponibles immédiatement 
mais susceptibles de le devenir assez rapidement. Ce compartiment est largement pourvu dans ce sol. Plusieurs études ont d'ailleurs montré la possibilité de plants forestiers à croître sur des sols «scalpés», sans réserves assimilables, jugées évidemment par les méthodes classiques d'analyse (WILNE \& YVER, 1962). Il n'empêche que ces bilans globaux font ressortir la relative fragilité de tels écosystèmes dans la mesure où, même si l'on ne s'adresse pas à la totalité des réserves réellement utilisables par les végétaux quand on parle d'éléments échangeables (ou assimilables), il s'agit des éléments qui sont le plus facilement mis à la disposition des plantes. L'exportation massive de la biomasse (surtout des petits compartiments, même sans prendre en compte les feuilles) ne pourra se faire longtemps sans restitution (fertilisation). On constate que l'exportation de ces petits compartiments est très consommatrice de bioéléments pour des produits qui n'ont pas actuellement une grande valeur économique. La dynamique et l'intensité des restitutions des éléments par altération n'est pas prise en compte. La mesure des flux entre complexe d'altération et éléments échangeables est capitale, mais nécessite la connaissance préalable des processus d'altération qui permettront d'évaluer les variations du compartiment «éléments échangeables».

\section{Conclusions}

Cette étude comparative de deux écosystèmes par l'approche globale apporte quelques résultats dans trois domaines :

1 - Ia distribution qualitative des bioéléments et son implication sur l'échantillonnage ;

2 - la distribution quantitative compartimentée des bioćléments dans les deux types de peuplements et des flux entre compartiments (immobilisation - restitution - prélèvements) ;

3 - influence d'une éventuelle substitution d'essence sur le système.

La complexité du système feuillu (taillis-sous-futaie) par rapport au système résineux (pessière équicnne) conduit à une multiplication des interventions pour un échantillonnage objectif, qui doit tenir compte des niveaux suivants :

- des strates quand elles existent (strate herbacée, taillis, futaie) :

- des espèces (les différences entre les espèces sont toujours significatives au moins pour un élément) ;

- des compartiments (pris généralement dans l'acceptation «organe», mais pouvant nécessiter des sous-divisions comme cette étude pour les branches des épicéas où la position relative dans la couronne ne doit pas être négligée);

- des distributions des arbres dans les différentes classes.

Dans l'état actuel de nos connaissances, il est nécessaire de renouveler de telles opérations qui permettront a posteriori d'optimiser l'échantillonnage. Pour évaluer les minéralomasses, il nous paraît préférable d'utiliser les lois de distribution des biomasses pour constituer des échantillons homologues, plutôt que de se servir de la notion « d'arbre moyen », qui ne correspond pas à la complexité des distributions des éléments minéraux dans les peuplements. 
Les principaux résultats de cette étude sont :

- l'établissement de tarifs de minéralomasse;

- l'évaluation à l'hectare apportant des données sur les immobilisations, donc sur les exportations en fonction du mode d'exploitation.

Létude du cycle biologique simplifié confirme la frugalité des peuplements forestiers : l'immobilisation courante dans la biomasse ligneuse est presque compensée par les apports atmosphériques (au moins pour la région industrialisée et la période étudiée) et elle est faible par rapport à la restitution. L'extrapolation à la duréc d'une révolution de futaic (150 ans) est sans doute plus significative. Pendant cette période, la production de la pessière est le double de celle du taillis-sous-futaie avec un rendement biologique très supérieur pour les éléments N, P, K et Ca. Les produits élaborés sont aussi très différents (l'essentiel de l'accroissement ligneux de la pessière se porte sur le bois de tronc). Les immobilisations des deux peuplements sont du même ordre que les stocks des éléments dits assimilables du sol (K, Ca, Mg, Mn). Selon l'intensité de la récolte, l'exportation peut être équivalente à l'immobilisation. Du point de vue bilan, l'écosystème peut être représenté schématiquement comme une boîte noire avec des entrées et des sorties.

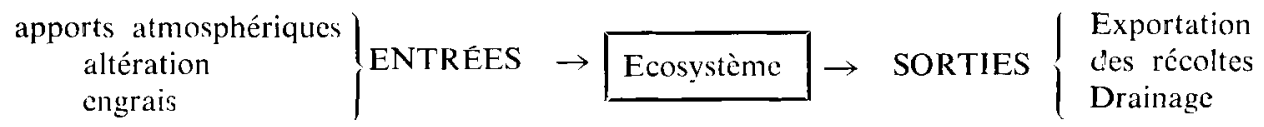

Lorsque les sorties excèdent les entrées le système se déséquilibre. II en résulte une baisse de production.

L'extrapolation à la durée d'une révolution de 150 ans de culture cxtensive conduit à la conclusion apparemment exagérée dun épuisement du compartiment des éléments assimilables. Il convient, avant de se prononcer définitivement, d'approfondir la signification du rapport Immobilisation/Réserves assimilables. Les plantes puisent dans ce compartiment des réserves assimilables, renouvelées par l'altération, qui compense au moins en partic l'effet du prélèvement. Il est donc primordial de connaittre le flux.

$$
\text { Réserves inaltérées } \rightarrow \text { Complexe d’altération } \rightarrow \text { Eléments assimilables }
$$

C'est seulement quand on aura progressé dans ce domaine que l'on pourra interpréter le rapport

$$
\text { Immobilisation totale }
$$

$$
\text { Eléments assimilables }
$$

et juger objectivement de l'impact de la récolte de la biomasse totale sur l'évolution de la fertilité du sol. D'ores et déjà il ressort que le rapport le plus significatif est le suivant :

$$
\text { Immobilisation courante }
$$

Eléments assimilables

Pour ces deux peuplements, les valeurs les plus fortes sont obtenues pour le potassium et le calcium, ainsi que pour le manganèse dans le cas de la pessière, indiquant un risque potentiel de déséquilibre. En contrepartie, les écosystèmes étudiés se révèlent performants vis-à-vis de l'utilisation des bioéléments et adaptés aux conditions de sols 
pauvres généralement réservés à la forêt. Sur les sols les plus pauvres, l'exportation des petits compartiments (branches) gros consommateurs de bioéléments est à déconseiller, sauf si le bilan économique permet leurs restitutions (engrais). C'est la position défendue à l'étranger (MälKöNEN, 1976 ; CAREY, 1980).

L'étude comparée du cycle biologique montre que la substitution d'essences feuillus résineux modifie sensiblement l'équilibre initial de la forêt feuillue, en agissant à la fois sur le cycle de la matière organique (Gulllaud, 1979 ; RANGER et al., 1981 a et b) et l'évolution du sol (Nys, 1981), ainsi que sur le cycle hydrologique (RICHERT, 1978; Nys et al., 1979) et le cycle biologique proprement dit.

Reçu pour publication le 13 avril 1982.

\section{Summary}

Comparative study of two forests ecosystems in french primary Ardennes. III. Bioelements content and biological cycle.

This paper gives the results of the comparative study of two forest ecosystems (a coppice with standards - 30 years old coppice and 150 years old standards, and a 50 years old spruce stand) from the bioelements content and biological cycle point of view.

The main results concern :

\section{Methodology}

Some rationalization of the sampling can be obtained. The variability of bioelements concentrations can be partially explained by the comparison (between compartments and species) and by the relationships with morphological parameters.

\section{Local estimation}

- compartmented bioelements content tables are built;

- data for the two stands are given. The data give, with sufficient accuracy, the bioelements drain caused by various harvesting methods (for the same type of stands in the same regions).

\section{Dynamics of the ecosystems}

Main annual flows between vegetation and soil are measured. Annual uptake (bioelements accumulation in the woody parts of the forest + soil return (litterfall + throughfall and stemflow) can be calculated). Annual bioelements accumulation is very similar in the two stands with a very different efficiency (Biomass/Bioelement content). Gross data were not directly comparable so we calculated bioelements accumulation during a more significant period : 150 years (one rotation for standards, five rotations for coppice and two rotations for spruce). Biomass production, mineral content and the evolution of soil fertility are discussed.

The results show that even of total bioelements accumulation can be partially replaced by precipitation input and weathering, the high extra-drain caused by slash utilization seems to be undesirable practice at least in poor soils.

\section{Références bibliographiques}

Atriwill, 1962. Estimations branch dry weight and leaf area from measurements of branch girth in Eucalyptus. Forest Science, 8 (2), 132-141.

BonNEAu et collaborateurs, 1977. Modification de fertilité des sols sous boisements artificiels de résineux purs. Compte rendu de fin d'étude de contrat D.G.R.S.T., tirage interne C.N.R.F., Sols forestiers. Distribution limitée.

BunN E.H., Will G.M., 1973. Influence des opérations d'aménagement sur le cycle des éléments fertilisants. Colloque international sur l'utilisation des engrais en forêt, publ. F.A.O./I.U.F.R.O., 35-56. 
Carey M.L., 1980. Whole tree harvesting in Sitka spruce : possibilities and implications. Irish forestry, 37 (1), 48-63.

Decourt N., Nys C., 1976. Nutrition, croissance et production de l'épicéa et du douglas dans le Limousin, R.F.F., XXVIII (3), 193-200.

Duvigneaud P., Paulet E., Kestemont P., Tanghe M., Denaeyer de Smet S., Schnock G., Timperman J., 1972. Productivité comparée d'une hêtraie (Fagetum) et d'une pessière (Picetum) établies sur même roche-mère à Mirwart (Ardennes luxembourgeoises). Bull. Soc. Roy. Bot. Belg., 105, 183-195.

Guillaud J., 1979. Etude comparée du cycle biogéochimique de l'azote dans deux écosystèmes forestiers feuillus et résineux des Ardennes primaires. Mémoire $3^{\circ}$ année E.N.I.T.E.F., 78 p. + annexes.

Katagiri Sh., Tsutsumi T., 1978. The relationships between site condition and circulation of nutrient in forest ecosystem (V). The difference in nutrient circulation between stand in upper part of slope and lower part of slope. J. Jap. For. Soc., 60, 195-202.

Le Tacon F., Oswald H., Tomassone R., 1970. La nutrition minérale de l'épicéa en Haute-Ardèche. Liaison avec la production. Ann. Sci. for., 27 (4), 357-381.

LEVY G., 1978. Nutrition et production de l'épicéa commun adulte sur sols hydromorphes en Lorraine : liaison avec les caractéristiques stationnelles. Ann. Sci. for., 35 (1), 33-53.

MADGWICK H.A.I., 1964. Variation in the chemical composition of red pine leaves : a comparison of well-grown and poorly grown trees. Forestry, 37 (1), 87-94.

MaLKonen E., 1976. The effect of fuller biomass harvesting on soil fertility. Symposium on the harvesting of a larger part of the forest biomass. 1, T. 1, Hyuin Kaa (Finlande) I.U.F.R.O./F.A.O./C.E.E./O.I.T.

Miller H.G., CoOper S.M., Miller J.D., 1976. Effect of nitrogen supply on nutrients in litter fall and crown leaching in a stand of Corsican Pine. J. appl. Ecol., 13, 233-248.

Morrisson I.K., 1972. Distribution of elements in aerial compoments of several Natural Jack Pine stands in Northern Ontario. Can. J. for. Res., 3, 170-179.

Nys C., 1981. Modifications des caractéristiques physico-chimiques d'un sol brun acide des Ardennes primaires par la monoculture d'épicéa commun. Ann. Sci. for., 38 (2), 237-258.

Nys C., Paternoster M., Vedy J.C., 1979. Transfert et redistribution des éléments en solution par les eaux de gravité de deux écosystèmes feuillu et résineux sur schistes du Révinien. Migrations organo-minérales dans les sols tempérés. Colloque, Nancy, 24-28 sept. 1979, publ. C.N.R.S.

Parde J., 1980. Problèmes de biomasses forestières énergétiques. Communication au Colloque international "Agriculture et Energie», C.E.N.E.C.A., Paris, 27, 28, 29 février 1980.

RANGER J., 1981. Etude de la minéralomasse et du cycle biologique dans deux peuplements de pin laricio de Corse dont l'un a été fertilisé à la plantation. Ann. Sci. for., 38 (1), 127-158.

RANGer J., Nys C., Ranger Dominique, 1981 a. Etude comparative de deux écosystèmes forestiers feuillu et résineux des Ardennes primaires françaises. I. - Biomasse aérienne du taillis-sous-futaie. Ann. Sci. for., 38 (2), 259-282.

Ranger J., Nys C., Ranger Dominique, 1981 b. II. - Biomasse aérienne d'une plantation équienne d'épicéa. Ann. Sci. for., 38 (3), 377-388.

Richert D., 1978. Etude de l'influence des résineux sur l'acidité des sols et des eaux. Mémoire $3^{e}$ année E.N.I.T.E.F., publ. C.N.R.F.

ULRICH B., 1973. Influence de la fertilisation sur le cycle des éléments nutritifs dans les écosystèmes forestiers. Colloque international sur l'utilisation des engrais en forêt, publ. F.A.O./I.U.F.R.O., 23-34.

VAN DEN DrIESSChe R., 1974. Prediction of mineral nutrient status of trees by foliar analysis. The Botanical Review, 40 (3), 347-388.

Wells C.G., Metz L.S., 1963. Variation in nutrient content of Loblolly pine needles with season, age, soil and position on the crown. Soil Sci. Am. Proc., 27, 90-93.

Wilde S.A., IYer J.G., 1962. Growth of red pine (Pinus resinosa Ait.) on scalped soils. Ecology, 63 (4), 771-774. 